\title{
Electronic phase engineering induced thermoelectric enhancement in manganites
}

Ping Jia, Yinlong Wang, Zhibo Yan, Jijun Gong, Lin Lin, Feng Gao and J-M Liu

The self-archived postprint version of this journal article is available at Linköping University Institutional Repository (DiVA):

http:// urn.kb.se/ resolve?urn=urn:nbn:se:liu:diva-150260

N.B.: When citing this work, cite the original publication.

Jia, P., Wang, Y., Yan, Z., Gong, J ., Lin, L., Gao, F., Liu, J ., (2018), Electronic phase engineering induced thermoelectric enhancement in manganites, J ournal of Applied Physics, 124(3), 034501. https:// doi.org/ 10.1063/ 1.5026868

Original publication available at:

https:// doi.org/ 10.1063/1.5026868

Copyright: AIP Publishing

http:// www.aip.org/ 


\title{
Electronic phase engineering induced thermoelectric enhancement in manganites
}

\author{
Ping Jia ${ }^{1}$, Yinlong Wang ${ }^{1}$, Zhibo Yan ${ }^{1,2}$ a), Jijun Gong ${ }^{1}$, Lin Lin $^{1}$, Feng Gao ${ }^{2}$, and J. -M. Liu ${ }^{1}$ \\ ${ }^{1}$ Laboratory of Solid State Microstructures and Innovation Center of Advanced \\ Microstructures, Nanjing University, Nanjing 210093, China \\ ${ }^{2}$ Biomolecular and Organic Electronics, IFM, Linköping University, Linköping, Sweden
}

\begin{abstract}
[Abstract] The nano-structuring engineering and the introduction of magnetic scattering are effective ways to enhance the thermoelectric performance. In this work, we use the magnetic treatments on $\mathrm{La}_{0.4} \mathrm{Pr}_{0.225} \mathrm{Ca}_{0.375} \mathrm{MnO}_{3}$ to demonstrate that the electronic phase engineering can enhance the thermoelectric performance by simultaneously reducing the thermal conductivity and raising the power factor in a strongly correlated electron system. The study indicates that the magnetic treatment changes the phase separation state and impedes the growth of ferromagnetic metal (FMM) phase. The decrease of FMM phase miniatures the FMM domain sizes and suppresses the bipolar effect, which raises the Seebeck coefficient and the power factor, reduces the thermal conductivity, and therefore enhances the thermoelectric performance.
\end{abstract}

Keywords: $\quad$ Thermoelectric, Seebeck effect, manganites, electric phase separation

a) Correspondent, E-mail: zbyan@nju.edu.cn 


\section{INTRODUCTION}

Developing high efficient thermoelectric technology has attracted much attention recently due to their applications in solid-state refrigeration and electrical generation from waste heat. $^{1,2}$ The thermoelectric efficiency is determined by the dimensionless figure of merit $Z T=\left(S^{2} \sigma / \kappa\right) T$, where $T$ is the absolute temperature, $S$ is the Seebeck coefficient, $\sigma$ is the electrical conductivity, and $\kappa$ is the thermal conductivity. The enhanced thermoelectric efficiency requires a conflicting combination of low $\kappa$, high $S$, and high $\sigma^{3}$ For example, the decrease of grain size raises the $S$ due to the quantum confinement effect, reduces the $\kappa$ because of the enhanced phonon scattering, but also reduces the $\sigma$ which limits the enhancement of $Z T{ }^{4}$ Besides, due to the thermoelectromagnetic effect, the embedding of soft magnetic nanoparticles in the thermoelectric matrix can introduce new carriers and multiple carrier scattering, which raises the $\sigma$ and the $S$ respectively. ${ }^{5}$ However, the challenge is that the magnetic atoms/ions in thermoelectric matrix easily lost its soft magnetic behavior due to the chemical binding with surrounding ions. Inspired from the above two methods, we study the electronic phase engineering for enhancing the ZT by simultaneously modulating the electronic phase domain and the magnetic scattering in a strongly correlated electron system.

In strongly correlated electron systems such as manganites, due to the strongly coupling of spin, orbital, charge, and lattice degree of freedoms, the involved electronic phase transition usually accompanies with a magnetic and a metal-insulator transition (MIT). Furthermore, the multiple electronic phases with different magnetic structures and electrical conductivity coexist in the system. ${ }^{6}$ As one of the coexisting phases in manganites, antiferromagnetic charge-ordered insulator (AFM/COI) phase is a result of super-exchange interaction in the $\mathrm{Mn}^{3+}-\mathrm{O}^{2-}-\mathrm{Mn}^{4+}$ configuration, by which the moments of $\mathrm{Mn}^{3+}$ and $\mathrm{Mn}^{4+}$ ions are antiparallel and the charge carriers are localized on the lattice. ${ }^{7}$ Another coexisting phase is the ferromagnetic metal (FMM) phase that is determined by the double-exchange interaction in the $\mathrm{Mn}^{3+}-\mathrm{O}^{2-}-\mathrm{Mn}^{4+}$ configuration. Due to the double-exchange interaction, the simultaneous transfer of an electron from $\mathrm{Mn}^{3+}$ to $\mathrm{O}^{2-}$ and from $\mathrm{O}^{2-}$ to $\mathrm{Mn}^{4+}$ causes a high electrical conductivity and a ferromagnetic correlation between $\mathrm{Mn}^{3+}$ and $\mathrm{Mn}^{4+}$ ions. ${ }^{7}$ Early investigations have revealed that the coexisting phases are controllable by electronic phase 
engineering. ${ }^{8}$ The engineering method includes chemical doping, thermal annealing, electrical and/or magnetic field treatment, etc. ${ }^{9-11,12}$

As a well investigated electron system, $\mathrm{La}_{3 / 8-y} \mathrm{Pr}_{\mathrm{y}} \mathrm{Ca}_{3 / 8} \mathrm{MnO}_{3}$ owns a martensitic-like electronic phase transition between the AFM/COI and the FMM phase. ${ }^{6,8}$ The martensitic-like phase transition allows for the coexistence of AFM/COI and FMM phase at nanometer or submicrometer scale and determines the formation of a electronic phase separation state. ${ }^{13,14}$ Due to the vivid competition between the coexisting phases, a small external stimulus can give a remarkable change of the electronic phase separation state. ${ }^{6,8,10}$ This can be utilized to engineer the electronic phases. ${ }^{15}$

$\mathrm{La}_{3 / 8-\mathrm{y}} \mathrm{Pr}_{\mathrm{y}} \mathrm{Ca}_{3 / 8} \mathrm{MnO}_{3}$ exhibits a percolation-type transport behavior. ${ }^{16}$ The FMM phase has a higher $\kappa$ and a higher $\sigma$ but a lower $S$ as compared with the AFM/COI phase. ${ }^{16}$ A small variation of phase separation state will obviously change the electrical and the thermal transport behaviors. In this work, we use magnetic treatments to demonstrate how the electronic phase engineering affects the thermoelectric properties of $\mathrm{La}_{0.4} \mathrm{Pr}_{0.225} \mathrm{Ca}_{0.375} \mathrm{MnO}_{3}$ (LPCMO). The result indicates that the magnetic treatment reduces the thermal conductivity, raises the Seebeck coefficient, and consequently enhances the thermoelectric performance.

\section{EXPERIMENTAL DETAILS}

High quality polycrystalline LPCMO bulk was synthesized by the standard solid-state reaction. No any impurity phase is observed within the X-ray diffraction detection limit. The magnetization was measured with 1000 Oe on a superconducting quantum interference device (SQUID). The resistivity, Seebeck coefficient, thermal conductivity, and Hall resistance were measured on a physical property measurement system (PPMS) with $2 \mathrm{~K} / \mathrm{min}$. The resistivity was measured by four-probe configuration with $20 \mu \mathrm{A}$. The Seebeck coefficient and the thermal conductivity were measured by the steady-state method on a standard thermal transport sample station. During the Seebeck coefficient measurement, the direction of temperature gradient is parallel to the magnetic field during the magnetic sweep, which avoids the influence from longitudinal Nernst effect. We measured the temperature-dependent Hall voltages: $V_{H 1}\left(T, B_{0}, I_{0}\right), V_{H 2}\left(T, B_{0},-I_{0}\right), V_{H 3}\left(T,-B_{0}, I_{0}\right)$, and $V_{H 4}\left(T,-B_{0},-I_{0}\right)$, where $B_{0}=0.5 T$ 
and $I_{0}=20 \mathrm{uA}$, then calculated the Hall resistance with $R_{H}=\left(V_{H 1}-V_{H 2}-V_{H 3}+V_{H 4}\right) /\left(4 * I_{0}\right)$.

Our previous work demonstrates that the cooling from room temperature transits the LPCMO from paramagnetic insulator (PMI) to AFM/COI phase at $200 \mathrm{~K}$ and then transits to FMM phase at $\sim 95 \mathrm{~K} .{ }^{9}$ The electronic phase separation state appears below $200 \mathrm{~K}$, and is predominated by the COI phase as $95 \mathrm{~K}<T<200 \mathrm{~K}$ while by the FMM phase as $T<95 \mathrm{~K} .{ }^{10}$ The following sequence is the magnetic treatment protocol:

1) Cool from room temperature to a treating temperature $T_{\text {treat }}\left(95 \mathrm{~K}<T_{\text {treat }}<200 \mathrm{~K}\right)$;

2) Apply a magnetic field sweep $\left(0 \rightarrow B_{\max } \rightarrow-B_{\max } \rightarrow 0\right)$ at $T_{\text {treat }}$;

3) Continue to cool down from $T_{\text {treat }}$ to $5 \mathrm{~K}$ without magnetic field;

4) Warm back to room temperature.

During these sequences, the Seebeck coefficient, the Hall resistance, the resistivity, the thermal conductivity, and the magnetism of LPCMO were measured respectively.

\section{RESULTS}

Figure 1(a) shows the temperature dependent Seebeck coefficient with and without the magnetic treatment $\left(B_{\max }=5 \mathrm{~T}\right)$ at $T_{\text {treat }}=145 \mathrm{~K}$ respectively. Without the magnetic field treatment, the Seebeck coefficient shows a hysteresis loop during the cooling and warming processes, consistent with the character of martensitic-like phase transition in LPCMO. ${ }^{8}$ The maximum Seebeck coefficient is about $65 \mu \mathrm{V} / \mathrm{K}$ at $\sim 81 \mathrm{~K}$. After the magnetic treatment, however, the Seebeck coefficient has a little decrease at $T_{\text {treat }}$, but quickly increases in the subsequent cooling and reaches to a peak of $\sim 260 \mu \mathrm{V} / \mathrm{K}$ at $\sim 77 \mathrm{~K}$, which is about 4 times larger than that without the treatment. With the further cooling, the Seebeck coefficient drops to a negative valley at $\sim 67 \mathrm{~K}$ before approaching to zero. This variation of Seebeck coefficient implies that LPCMO is a highly compensated system in which both the hole-like and electron-like carriers are contributing to the thermoelectric properties.

Figure 1(b) indicates that the magnetic treatment raises the resistivity and lowers down the MIT temperature to $\sim 75 \mathrm{~K}$ where the Seebeck coefficient reaches to the maximum. After the magnetic treatment, the MIT peak is raised from $8.33 * 10^{4} \Omega \cdot \mathrm{cm}$ to $1.63 * 10^{5} \Omega \cdot \mathrm{cm}$, and the resistivity at $5 \mathrm{~K}$ increases from $4.75 \Omega \cdot \mathrm{cm}$ to $390 \Omega \cdot \mathrm{cm}$. Figure 2(a) shows the 
temperature dependent power factor $\left(P F=S^{2} \sigma\right)$, calculated from the data in Figure 1(a) and 1(b). The magnetic treatment at $T_{\text {treat }}=145 \mathrm{~K}$ with $B_{\max }=5 \mathrm{~T}$ obviously raises the power factor at the temperature between $70 \mathrm{~K}$ and $92 \mathrm{~K}$. The power factor near $77 \mathrm{~K}$ is about 0.042 $\mathrm{nWm}^{-1} \mathrm{~K}^{-2}$, which is more than 10 times larger than that without the magnetic treatment. Besides, Figure 2(b) shows that the magnetic treatment obviously reduces the thermal conductivity below $92 \mathrm{~K}$. Therefore, according to $Z T=(P F / \kappa) T$, the magnetic treatment remarkably enhances the $Z T$ between $70 \mathrm{~K}$ and $92 \mathrm{~K}$. Note that due to the martensitic-like phase transition, the variation of temperature sweep rate can affect the evolution of phase separation state and consequently affect the final $Z T$ value, but does not change this variation tendency.

We also study how the magnetic treatments with different $B_{\max }$ and $T_{\text {treat }}$ influence the Seebeck coefficient. Figure 5(a) shows the temperature-dependent Seebeck coefficient after the magnetic treatments at $T_{\text {treat }}=145 \mathrm{~K}$ with $B_{\max }=0,1 \mathrm{~T}, 2.3 \mathrm{~T}$, and $5 \mathrm{~T}$ respectively. With the increase of $B_{\max }$ from $1 \mathrm{~T}$ to $5 \mathrm{~T}$, the peak of Seebeck coefficient near 77K increases [shown in the inset of Figure 5(a)] and the valley of that near 67K becomes more obviously. Besides, Figure 5(b) shows the temperature dependent Seebeck coefficient after the magnetic treatment with $B_{\max }=5 \mathrm{~T}$ at the AFM/COI phase dominated temperature region ( $\left.120 \mathrm{~K} \leq T_{\text {treat }} \leq 160 \mathrm{~K}\right)$. The Seebeck coefficient has a largest peak value of $\sim 260 \mu \mathrm{V} / \mathrm{K}$ and a most obvious negative valley as $T_{\text {treat }} \sim 145 \mathrm{~K}$. The inset of Figure 5(b) shows that the maximum Seebeck coefficient strongly depends on the $T_{\text {treat }}$ and the best $T_{\text {treat }}$ is $\sim 145 \mathrm{~K}$. This data indicates that the magnetic treatment with different $T_{\text {treat }}$ and $B_{\max }$ can well tune the Seebeck coefficient.

\section{DISCUSSIONS}

The magnetic sweep at $\boldsymbol{T}_{\text {treat }}$ changes the phase separation state. Starting from the initial phase separation state (obtained after the cooling from room temperature to $T_{\text {treat }}$ ), both the resistivity and the Seebeck coefficient are firstly decreased and then re-increased while the variation of magnetism is on the contrary during the forward $(0 \rightarrow 5 \mathrm{~T})$ and the backward $(5 \mathrm{~T} \rightarrow 0)$ magnetic sweeps, as shown in Figure 3 (a), (b), and (c) respectively. This is because the AFM/COI domains are transited to FMM phase under the magnetic field and then 
re-nucleated as the magnetic field is reduced, which changes the volume of FMM phase. ${ }^{14}$ After the magnetic sweep, however, both the resistivity and the magnetism are larger than that of the initial state while the Seebeck coefficient is smaller than that of the initial state, which indicates the formation of a new phase separation state. The previous magnetic force microscope (MFM) and Lorentz microscope observations have evidenced that a magnetic sweep changes the phase separation state. ${ }^{6,8}$

Due to the martensitic-like phase transition from FMM phase matrix, it is reasonable that the new phase separation state has a little larger magnetism than the initial state, shown in the inset of Figure 3(b). In the usual situation, a larger magnetism means a smaller resistance due to the properties of FMM phase. However, the new phase separation state has an abnormal higher resistivity than the initial state, which maybe origin from the following two reasons. One is the variation of the phase domain distributions, which plays a crucial role on the electrical and thermal transport properties in a percolation system. ${ }^{17}$ The other is the relaxation of accommodation strain, which impedes the growth of AFM/COI phase but gives rise to complex phase domain boundaries possibly composed by charge-disordered spin-glass (CD-SG) insulator or spin-canted insulator phases. ${ }^{6,8,12,14}$ The spin-canted insulator state has a high resistivity but exhibits a little larger magnetic moment than the AFM/COI phase because of the spin canting. ${ }^{7,18}$

The new phase separation state impedes the growth of FMM phase and reduces the thermoelectric bipolar effect. Figure 4(a) shows the temperature-dependent magnetism with and without the magnetic treatment with $B_{\max }=5 \mathrm{~T}$ at $145 \mathrm{~K}$ respectively. After the magnetic treatment, the increase of magnetism in the subsequent cooling becomes slower, and the magnetism at $5 \mathrm{~K}$ decreases from $0.27 \mu_{\mathrm{B}} / \mathrm{Mn}$ to $0.10 \mu_{\mathrm{B}} / \mathrm{Mn}$. Besides, the temperature-dependent resistivity obviously increases after the magnetic treatment [shown in Figure 1(b)]. Therefore, the new phase separation state at $T_{\text {treat }}$, obtained after the magnetic treatment, impedes the growth of FMM phase during the cooling process. This maybe because the CD-SG or spin-canted insulator phase in the new phase separation state cannot be transited to the FMM phase and only a reduced AFM/COI phase is transited to the FMM phase in the cooling process. The CD-SG or spin-canted insulator phase is the possible stable 
state at low temperature. ${ }^{6,8,12,14}$

In an inhomogeneous magnetic system like LPCMO, the carriers will be driven to move in a direction perpendicular to both the current and the magnetic field by the spin-dependent scattering or by the Berry phase effect (the exact mechanism is still in debate), named as anomalous Hall effect. ${ }^{7,15,19}$ Viewing from the ordinary Hall effect, however, the electrons/holes may be moved to a "wrong” side. For a phenomenological understanding of Hall resistance, the "wrongly-moved" electrons and the "rightly-moved" holes are marked as hole-like carriers, while the "wrongly-moved" holes and the "rightly-moved" electrons are marked as electron-like carriers. With this treatment, the sign of Hall resistance indicates the dominance of electron-like or hole-like carriers, and the increase of Hall resistance suggests the decrease of electron-like or hole-like carrier concentration.

What is interesting is that the new phase separation state dramatically changes the temperature dependent Hall resistance, as shown in Figure 1(c). Without the magnetic treatment, the Hall resistance is almost temperature-independent and approaches to zero. After the magnetic treatment, however, the Hall resistance displays a positive peak at $~ 80 \mathrm{~K}$, then drops down to a negative valley at $\sim 67 \mathrm{~K}$, and finally approaches to zero below $50 \mathrm{~K}$.

The Hall resistance changed from a positive value to a negative one indicates a transformation from hole-like to electron-like carrier type. The temperature at which the Hall resistance change their sign is as the same as the MIT temperature $(\sim 75 \mathrm{~K})$, indicating that the MIT is accompanied by a carrier-type transformation. Therefore, the reduction of FMM phase means the decrease of the electron-like carriers. Besides, the magnetic treatment induced increase of resistivity implies the decrease of the averaged carrier concentration. ${ }^{19}$ With the decrease of carrier concentration, the amplitude of Hall resistance increases and the hole-like to electron-like carrier transformation at the MIT temperature naturally induces a dramatic variation of Hall resistance from a positive peak to a negative valley.

In Figure 1(c), the Hall resistance at the negative valley $(\sim-7.8 \mathrm{k} \Omega)$ is much larger in amplitude than that at the positive peak $(\sim 4.2 \mathrm{k} \Omega)$. This suggests that the magnetic treatment reduces the electron-like carriers much more than the hole-like carriers, inferred by supposing that only the hole-like/electron-like carriers are above/below the MIT temperature respectively. 
Due to the bipolar effect in thermoelectric, the Seebeck coefficients contributed by electron-like carriers and hole-like carriers cancel each other out. ${ }^{20}$ After the magnetic treatment, the much more reduction of the electron-like carriers than the hole-like carriers weakens the bipolar effect and hence enhances the positive Seebeck coefficient.

To further evidence that the new separation state at $T_{\text {treat }}$ weakens the thermoelectric bipolar effect, we measured the temperature-dependent Seebeck coefficient under the application of a constant magnetic field. Figure 4(b) shows that the positive Seebeck coefficient becomes smaller and smaller, and finally approaches to zero as the magnetic field increases from 0 to 5T. The holding on of a higher magnetic field will give the system a larger FMM phase and thus provide the more electron-like carriers, which sets the system at a more highly compensated status and leads to a smaller positive Seebeck coefficient. Therefore, the new separation state at $T_{\text {treat }}$ impedes the growth of FMM phase and reduces the bipolar effect, which maybe a major reason for the enhancement of Seebeck coefficient and power factor.

The new phase separation state possibly reduces the FMM domain sizes. The new phase separation state impedes the growth of FMM phase, which possibly leads to the decrease of FMM domain sizes. LPCMO is a percolated magnetic system having a high-resistivity matrix. The asymmetric scattering of spin-polarized current carriers at the domain boundaries has a substantial effect to the magnitude and the sign of Hall coefficient. $^{7,21}$ The decrease of FMM domain sizes would increase the skewness of carrier motion as the carriers travel in multiple collisions with the FMM boundaries, and consequently raise the Hall resistance by several orders of magnitude near the percolation threshold, ${ }^{7,21,22}$ which is consistent with the data in Figure 1(c). Besides, similar to the nano-structuring engineering of the crystalline grains, the decrease of FMM domain sizes would reduce the thermal conductivity through the enhanced phonon scattering and affect the Seebeck coefficient arising from quantum confinement effect. ${ }^{2,4,23}$ Therefore, the decrease of FMM domain sizes near the MIT temperature is another possible reason for the enhancement of thermoelectric properties.

The new phase separation state controls the thermoelectric properties. In Figure 5(a), the magnetic treatment with a larger $B_{\max }$ leads to a larger Seebeck coefficient. This is because 
a larger

At $\sim 77 \mathrm{~K}$, which is just a little higher than the MIT temperature ( 75K), the system has a small average carrier concentration and the dominated carrier-type is not transformed from hole-like to electron-like, which minimizes the bipolar effect and therefore causes to a highest Seebeck coefficient and a highest power factor.

A larger magnetic field change the phase separation state more seriously, because the AFM/COI phase in LPCMO begins to melt as $B>1 \mathrm{~T}$ and is completely transits to FMM phase when $B=5 \mathrm{~T}$. Furthermore, the FMM and AFM/COI phases dominate at different temperature regions and thus the magnetic treatment at different $T_{\text {treat }}$ will affect the final variation of phase separation state. These data indicate that the enhancement of Seebeck coefficient strongly depends on the variation of phase separation state.

During a magnetic sweep, a larger $B_{\max }$ implies a more complete relaxation of accommodation strain, because a larger magnetic field would erase the phase separation state more completely through the COI-to-FMM phase transition. Cooling from a new phase separation state with less accommodation strain, the growth of FMM phase will be weakened more seriously and result in a larger Seebeck coefficient according to the above analysis.

It is well known that the magnetism of AFM phase decreases with the temperature. The minimum of magnetism in Figure 4(a) implies that the AFM/COI phase is most stable or has a largest volume at $\sim 145 \mathrm{~K}$. A magnetic sweep with $B_{\max }=5 \mathrm{~T}$ at $145 \mathrm{~K}$ means the most serious destruction of AFM/COI structure before forming the new phase separation state, which greatly weakens the growth of FMM phase and hence produces a largest Seebeck coefficient during the subsequent cooling. This result further confirms that the magnetic treatment destroys the AFM/COI phase and thereafter impedes the growth of FMM phase in the subsequent cooling.

\section{CONCLUSION}

In summary, the electronic phase engineering on LPCMO is carried out through the 
magnetic treatment at $T_{\text {treat }}\left(120 \mathrm{~K} \leq T_{\text {treat }} \leq 160 \mathrm{~K}\right)$. The magnetism and resistivity measurements indicate that the magnetic treatment changes the phase separation state at $T_{\text {treat }}$ and impedes the growth of FMM phase in the subsequent cooling. The decrease of FMM phase suggests the decrease of the FMM domain sizes. By analogy with the nano-structuring engineering of crystalline, the decrease of FMM domain sizes is a possible reason for raising the Seebeck coefficient and reducing the thermal conductivity. Besides, the Seebeck coefficient and the Hall resistance measurements indicate that the insulator-to-metal transition accompanies with a hole-like to electron-like carrier transformation. After the magnetic treatment, the electron-like carriers are reduced much more than the hole-like carriers, which weakens the bipolar effect and hence raises the positive Seebeck coefficient and the power factor. This study demonstrates that the engineering of electronic phases is an effective way to enhance the thermoelectric performance in the strongly correlated systems.

\section{Acknowledgement:}

This work was supported by the National Key Research Programme of China (Grant Nos. 2016YFA0300101, 2016YFA0201004), the National Natural Science Foundation of China (Grant Nos. 51431006, 11374112), and the Fundamental Research Funds for the Central Universities (Grant No. 020414380063). 


\section{Caption of Figures:}

Figure 1. Temperature dependent (a) Seebeck coefficient, (b) resistivity, and (c) Hall resistance of LPCMO, with and without the magnetic treatment $(0 \rightarrow 5 \mathrm{~T} \rightarrow-5 \mathrm{~T} \rightarrow 0)$ at $T_{\text {treat }}=145 \mathrm{~K}$, respectively.

Figure 2. Temperature dependent (a) Power factor and (b) Thermal conductivity of LPCMO, with and without the magnetic treatment at $T_{\text {treat }}=145 \mathrm{~K}$, respectively.

Figure 3. Magnetic field dependent (a) Resistivity, (b) Magnetism, and (c) Seebeck coefficient of LPCMO after cooling the sample from $300 \mathrm{~K}$ to $145 \mathrm{~K}$. The inset is the local magnification of (b).

Figure 4. (a) Temperature dependent magnetism of LPCMO without the treatment $\left(B_{\max }=0\right)$ and with the treatment $\left(B_{\max }=5 \mathrm{~T}\right)$ at $T_{\text {treat }}=145 \mathrm{~K}$ respectively, measured with 1000 Oe. (b) Temperature dependent Seebeck coefficient of LPCMO, measured under a magnetic field of 0, 1T, 2.3T, and 5T respectively.

Figure 5. (a) Temperature dependent Seebeck coefficient, measured after the different magnetic treatments at $T_{\text {treat }}=145 \mathrm{~K}$, with $B_{\max }=0,1 \mathrm{~T}, 2.3 \mathrm{~T}$, and $5 \mathrm{~T}$ respectively. (b) Temperature dependent Seebeck coefficient, measured after a magnetic treatment $\left(B_{\max }=5 \mathrm{~T}\right)$ at $T_{\text {treat }}=125 \mathrm{~K}, 140 \mathrm{~K}, 145 \mathrm{~K}$, and $153 \mathrm{~K}$ respectively. 


\section{References:}

J. Zhang, L. Song, G. K. Madsen, K. F. Fischer, W. Zhang, X. Shi, and B. B. Iversen, Nat. Commun. 7, 10892 (2016); L. Li, Y. Liu, J. Y. Dai, A. J. Hong, M. Zeng, Z. B. Yan, J. Xu, D. Zhang, D. Shan, S. L. Liu, Z. F. Ren, and J. M. Liu, Journal of Materials Chemistry C 4, 5806 (2016).

2 G. Pennelli, Beilstein J Nanotechnol 5, 1268 (2014).

G. J. Snyder and E. S. Toberer, Nat. Mater. 7, 105 (2008).

Paothep Pichanusakorn and Prabhakar Bandaru, Materials Science and Engineering: R: Reports 67, 19 (2010); Bed Poudel, Qing Hao, Yi Ma, Yucheng Lan, Austin Minnich, Bo Yu, Xiao Yan, Dezhi Wang, Andrew Muto, Daryoosh Vashaee, Xiaoyuan Chen, Junming Liu, Mildred S. Dresselhaus, Gang Chen, and Zhifeng Ren, Science 320, 634 (2008); Wenwen Zhou, Jixin Zhu, Di Li, Huey Hoon Hng, Freddy Yin Chiang Boey, Jan Ma, Hua Zhang, and Qingyu Yan, Adv. Mater. 21, 3196 (2009).

W. Zhao, Z. Liu, Z. Sun, Q. Zhang, P. Wei, X. Mu, H. Zhou, C. Li, S. Ma, D. He, P. Ji, W. Zhu, X. Nie, X. Su, X. Tang, B. Shen, X. Dong, J. Yang, Y. Liu, and J. Shi, Nature 549, 247 (2017).

Y. Murakami, H. Kasai, J. J. Kim, S. Mamishin, D. Shindo, S. Mori, and A. Tonomura, Nature Nanotech. 5, 37 (2010).

Naoto Nagaosa, Jairo Sinova, Shigeki Onoda, A. H. MacDonald, and N. P. Ong, Rev. Mod. Phys. 82, 1539 (2010).

H. Zhou, L. Wang, Y. Hou, Z. Huang, Q. Lu, and W. Wu, Nat. Commun. 6, 8980 (2015).

Z. B. Yan and J. M. Liu, Applied Physics a-Materials Science \& Processing 104, 471 (2011).

L. Ghivelder and F. Parisi, Phys. Rev. B 71, 184425 (2005).

G. Singh-Bhalla, S. Selcuk, T. Dhakal, A. Biswas, and A. Hebard, Phys. Rev. Lett. 102, 077205 (2009); Y. Zhu, K. Du, J. Niu, L. Lin, W. Wei, H. Liu, H. Lin, K. Zhang, T. Yang, Y. Kou, J. Shao, X. Gao, X. Xu, X. Wu, S. Dong, L. Yin, and J. Shen, Nat. Commun. 7, 11260 (2016).

J. Q. He, V. V. Volkov, T. Asaka, S. Chaudhuri, R. C. Budhani, and Y. Zhu, Phys. Rev. B 82, 224404 (2010).

Z. B. Yan, K. F. Wang, S. Z. Li, S. J. Luo, and J. M. Liu, Appl. Phys. Lett. 95, 143502 (2009); K. H. Ahn, T. Lookman, A. Kryszczyńska, and A. R. Bishop, Nature 428, 400 (2004).

Weida Wu, Casey Israel, Namjung Hur, Soonyong Park, Sang-Wook Cheong, and Alex de Lozanne, Nature Mater. 5, 881 (2006).

M. B. Salamon and M. Jaime, Rev. Mod. Phys. 73, 583 (2001).

K. H. Kim, M. Uehara, C. Hess, P. A. Sharma, and S. W. Cheong, Phys. Rev. Lett. 84, 2961 (2000).

D. Khomskii and L. Khomskii, Phys. Rev. B 67, 052406 (2003).

Y. Tokura, Rep. Prog. Phys. 69, 797 (2006).

A. Y. Shik, Electronic properties of inhomogeneous semiconductors. (Gordon and Breach, Luxembourg ; United States, 1995), pp.xvii.

J. J. Gong, A. J. Hong, J. Shuai, L. Li, Z. B. Yan, Z. F. Ren, and J. M. Liu, Phys. Chem. Chem. Phys. 18, 16566 (2016).

A. V. Vedyaev, A. B. Granovskii, A. V. Kalitsov, and F. Brouers, J Exp Theor Phys+ 85, 1204 (1997).

Peng Xiong, Gang Xiao, J. Q. Wang, John Q. Xiao, J. Samuel Jiang, and C. L. Chien, Phys. Rev. Lett. 69, 3220 (1992).

Weishu Liu, Chuan Fei Guo, Mengliang Yao, Yucheng Lan, Hao Zhang, Qian Zhang, Shuo Chen, Cyril P. Opeil, and Zhifeng Ren, Nano Energy 4, 113 (2014). 


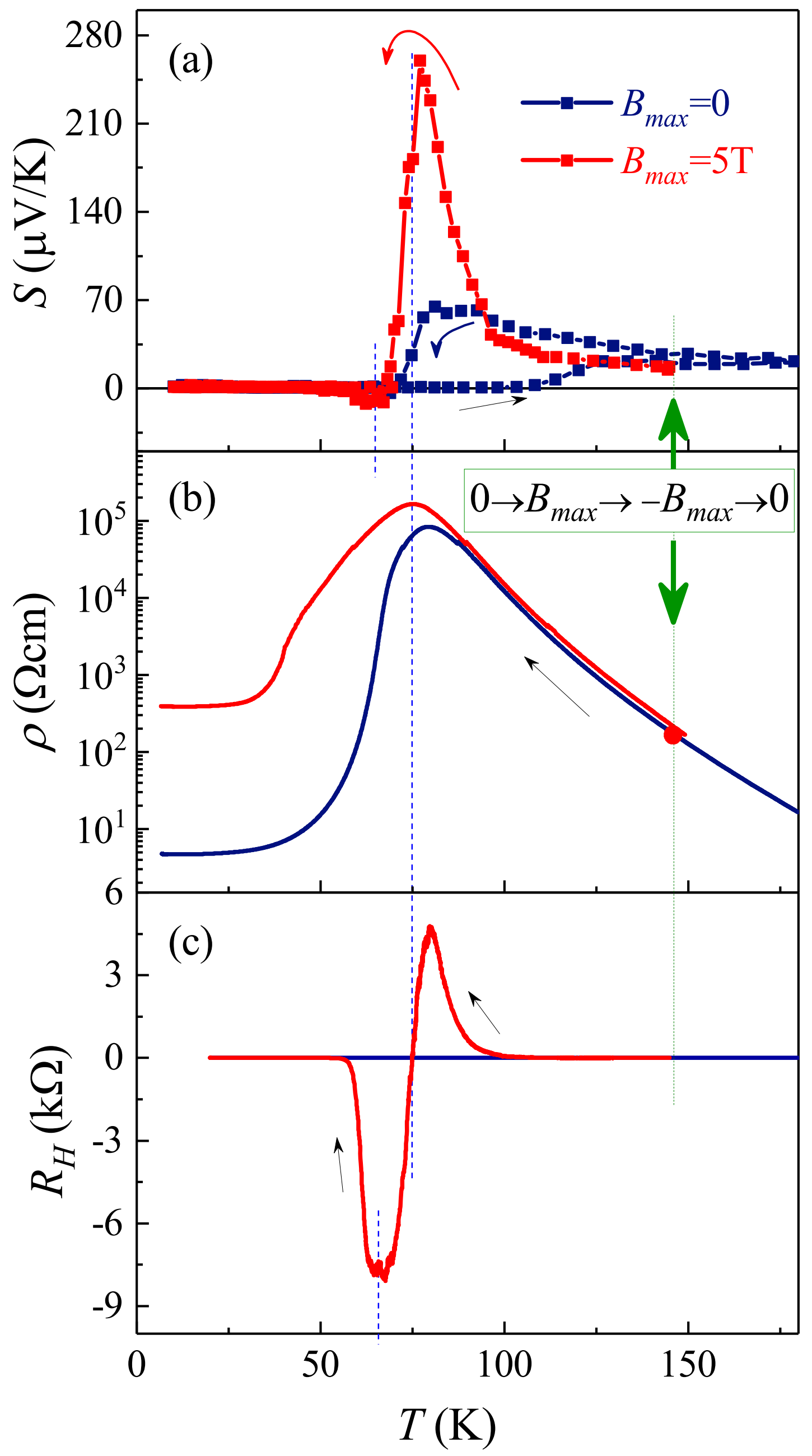



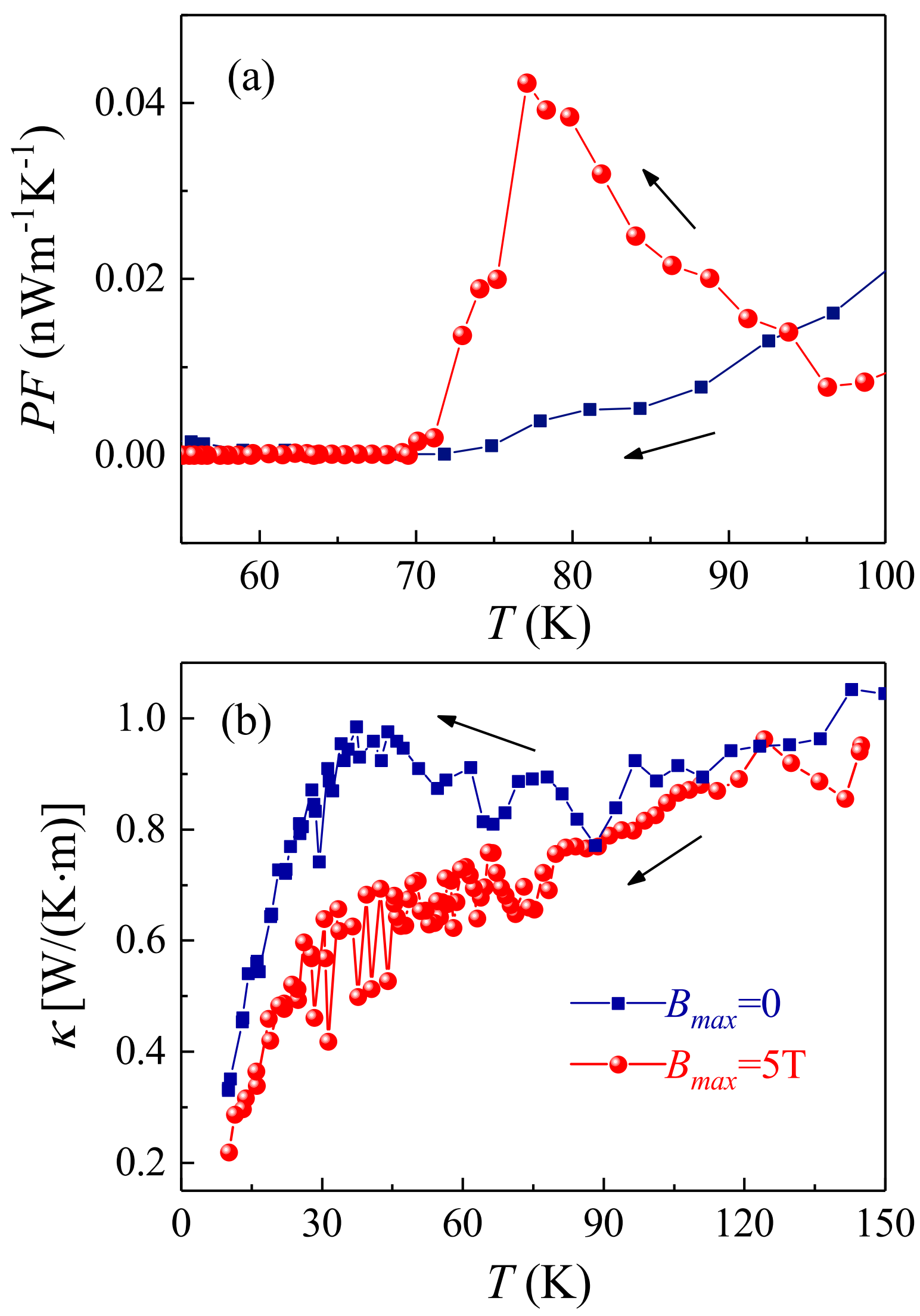


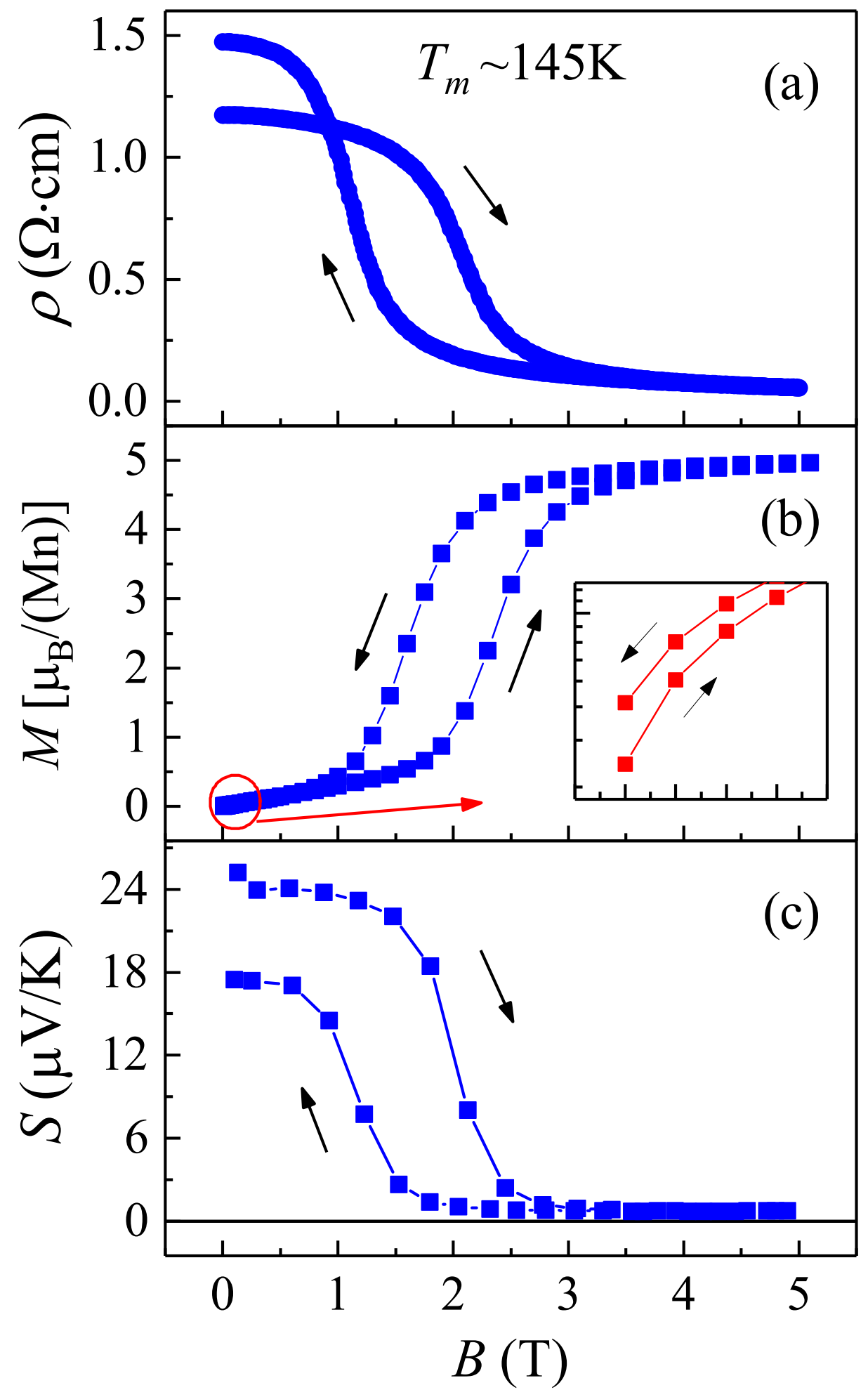



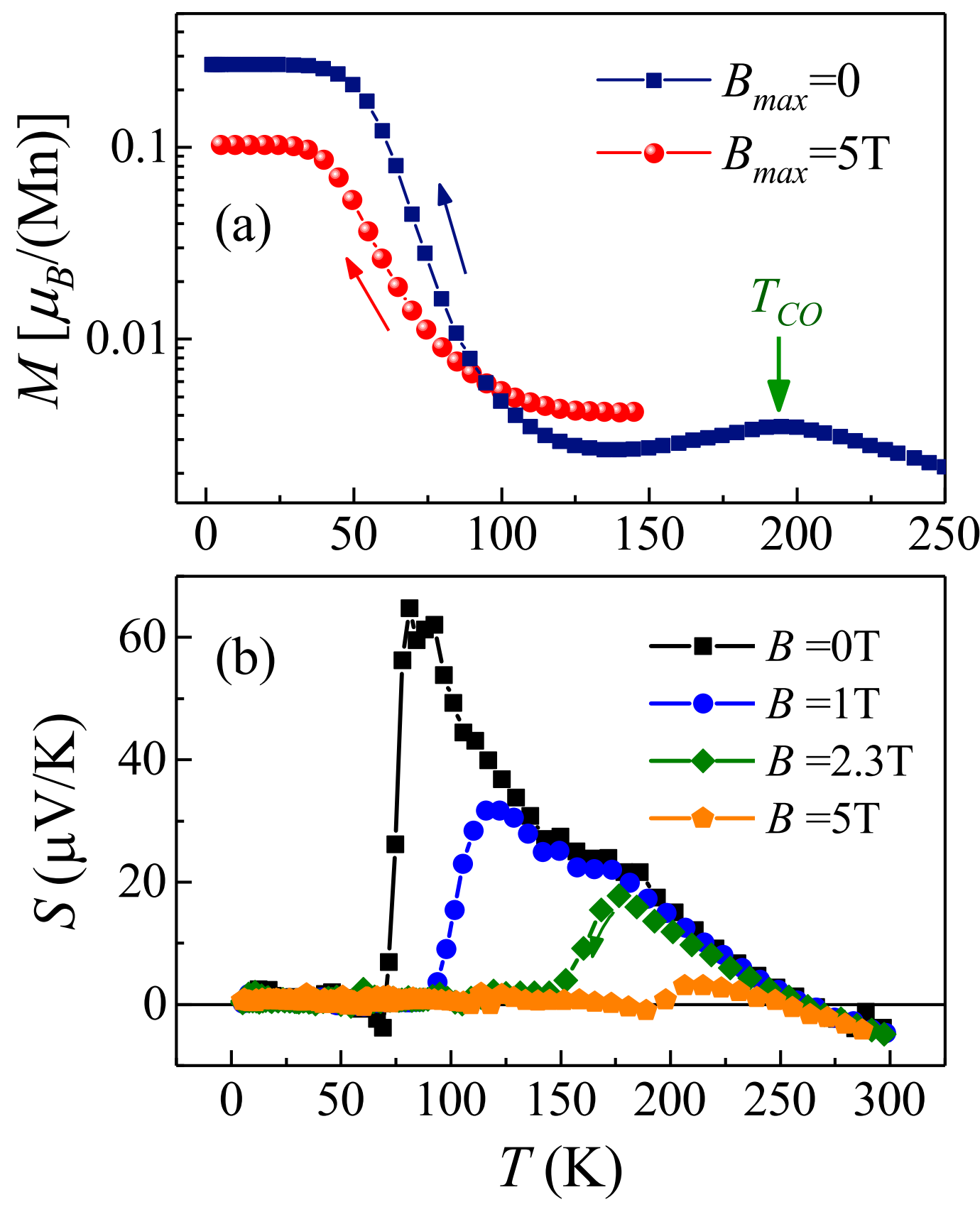

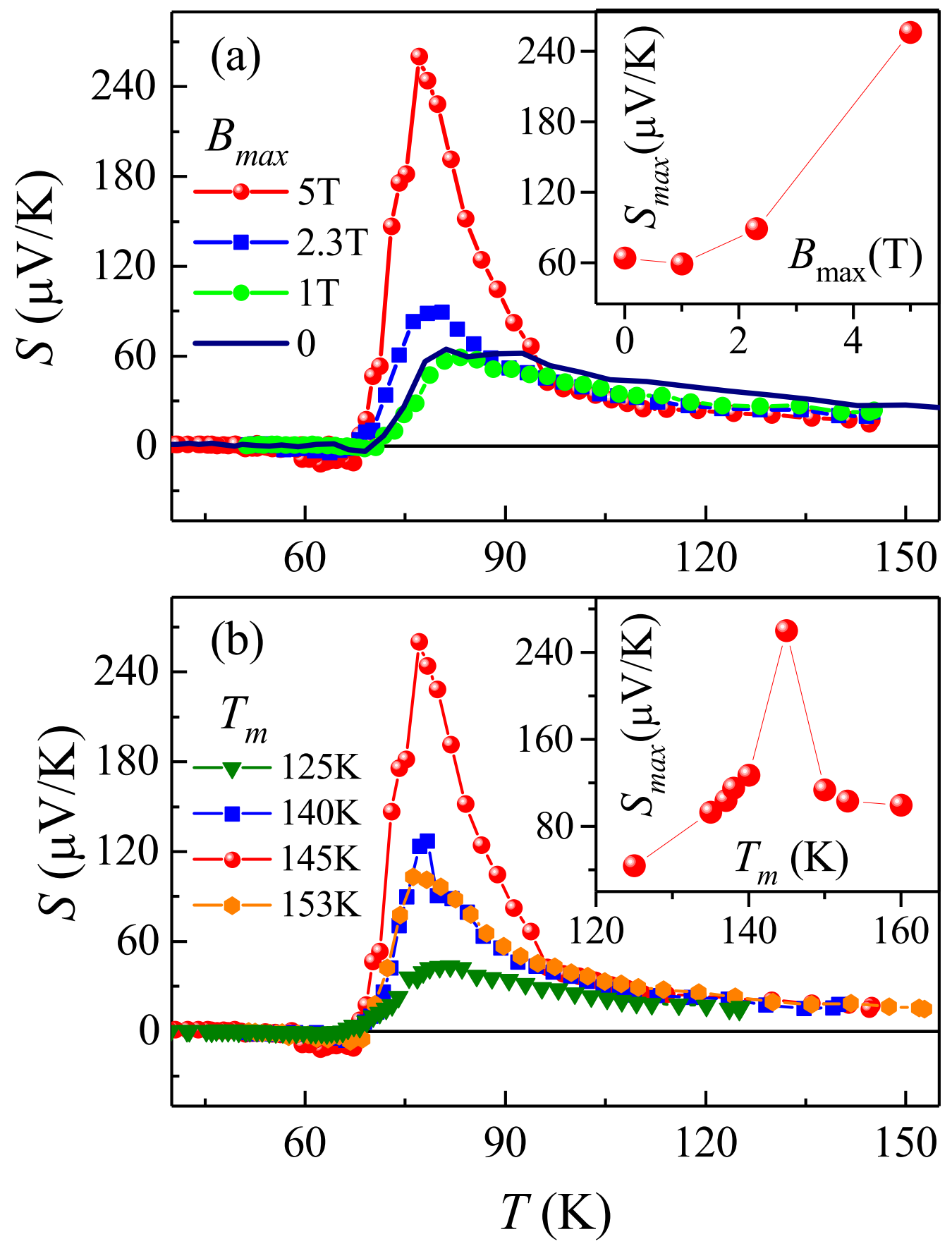